\title{
The location, histologic type, and stage of lung cancer are associated with bleeding during endobronchial biopsy
}

This article was published in the following Dove Press journal:

Cancer Management and Research

\author{
Saibin Wang ${ }^{1,2}$ \\ Qian $\mathrm{Ye}^{3}$ \\ Junwei Tu' \\ Yong Song ${ }^{2}$ \\ 'Department of Respiratory Medicine, \\ Jinhua Municipal Central Hospital, \\ Jinhua, Zhejiang Province, China; \\ ${ }^{2}$ Department of Respiratory Medicine, \\ Jinling Clinical Medical College of \\ Nanjing Medical University, Nanjing, \\ China; ${ }^{3}$ Department of Medical \\ Records Quality Management, Jinhua \\ Municipal Central Hospital, Jinhua, \\ Zhejiang Province, China
}

Background: Several risk factors have been proposed for bleeding during bronchoscopy, including immunosuppression, thrombocytopenia, pulmonary arterial hypertension, and mechanical ventilation. However, research on bronchoscopic biopsy-induced bleeding in the population of lung cancer without these "proposed risk factors" remains lacking.

Patients and methods: A total of 531 lung cancer patients with endobronchial biopsy (EBB) were enrolled in this retrospective observational study. Patients were divided into biopsy-induced bleeding group $(n=162)$ and non-bleeding group $(n=369)$. Using multiple logistic regression, independent risk factors for EBB bleeding were identified.

Results: The location, histologic type, and stage of lung cancer were independently associated with EBB bleeding, as assessed by multiple logistic regression $(p<0.05)$ in patients with lung cancer. Moreover, during EBB, the risk of bleeding of endobronchial lesions located in the central airways was significantly higher when compared to that in peripheral bronchi (odds ratio $[\mathrm{OR}], 2.211 ; 95 \% \mathrm{CI}, 1.276-3.830 ; p=0.005)$. In addition, squamous cell carcinoma and small-cell lung carcinoma were more susceptible to bleeding during biopsy when compared with adenocarcinoma (OR, 3.107, 2.389; 95\% CI, 1.832-5.271, 1.271-4.489; $p=0.000, p=0.007$, respectively). Patients with advanced lung cancer were more prone to EBB bleeding compared to patients in the early stages of disease (OR, 1.583; 95\% CI, 1.065-2.354; $p=0.023$ ).

Conclusion: Lesions located in the central airways, histologic types of squamous cell carcinoma and small-cell lung carcinoma, and stages of advanced lung cancer were the independent risk factors for hemorrhage in EBB.

Keywords: bronchoscopy, biopsy, hemorrhage, risk factor

\section{Introduction}

For bronchoscopists, hemorrhage is a frequently encountered and difficult-tomanage complication. ${ }^{1-3}$ Reported risk factors for bleeding during bronchoscopy include immunosuppression, mechanical ventilation, thrombocytopenia (platelets $<50 \times 10^{3} / \mu \mathrm{L}$ ), pulmonary arterial hypertension, lung transplant, anticoagulant and antiplatelet therapy, liver and kidney disease, bleeding tendencies and active bleeding. ${ }^{4-7}$ Fortunately, most patients who undergo bronchoscopy do not present these risk factors in clinical practice.

Bronchoscopic biopsies are widely used in the histopathologic diagnosis of lung cancer, which is one of the leading cancers worldwide causing morbidity and mortality. ${ }^{8}$ Common biopsy procedures include endobronchial forceps biopsy, cryobiopsy, bronchial brushing, and transbronchial needle aspiration. ${ }^{9,10}$ Malignancies may be more susceptible to hemorrhage compared to benign mucosal lesions and, therefore, 
require more attention during bronchoscopy. ${ }^{11-13}$ However, the risk factors of bronchoscopic biopsy-induced bleeding in patients with lung cancer remain elusive.

The aim of this study was to assess bronchoscopic biopsyinduced bleeding in patients diagnosed with lung cancer but without "proposed risk factors" and to identify independent risk factors for endobronchial biopsy (EBB) bleeding in this patient population.

\section{Patients and methods}

\section{Study design and data collection}

This retrospective cohort study was performed in patients with EBB who were diagnosed with lung cancer at the Jinhua Hospital of Zhejiang University between January 2014 and July 2017. The study was approved by the ethics committee of Jinhua Hospital of Zhejiang University (No. 2017102001). Relevant medical records, laboratory results, and histopathologic data collected from study subjects were anonymous, and informed consent was, therefore, waived.

The following data were extracted: age, gender, comorbidities (hypertension, diabetes, COPD, and heart disease), location of the lesion, histologic type of lung cancer, stage of cancer, biopsy results (bleeding or not), and methods of hemostasis maneuvers. Blood tests were performed within 72 hours prior to bronchoscopy and included white blood cell counts, neutrophils, C-reactive protein, hemoglobin, platelet counts, prothrombin time, activated partial thromboplastin time, alanine aminotransferase, and aspartate aminotransferase.

Patients were divided into two groups. Subjects who had been treated with hemostasis maneuvers during EBB were categorized under the bleeding group and the remaining subjects with no bleeding or minimal bleeding (bleeding stopped on its own) were included in the non-bleeding group. Moreover, based on their TNM stage, patients were categorized into early and advanced stages (TNM stage I and II as early stage and stage III and IV as advanced stage). In this study, trachea, left and right main bronchi, and the right middle bronchus were defined as the central airways. Both the upper lobar bronchi, the lower lobar bronchus, and the right middle lobar bronchus were classified as peripheral bronchi.

Patients' inclusion criteria were as follows: 1) adult patients with endobronchial local exophytic lesions who underwent EBB and 2) patients diagnosed with primary lung cancer based on histologic confirmation. Patients who presented any of the following conditions were excluded from the study: immunosuppression, mechanical ventilation, thrombocytopenia (platelets $<50 \times 10^{3} / \mu \mathrm{L}$ ), international normalized ratio $>1.3$, continuous anticoagulant or antiplatelet therapy, pulmonary arterial hypertension, superior vena cava syndrome, heart function failure, liver or kidney disease, lung transplant, active bleeding, known bleeding tendencies, or a suspected bleeding disorder.

\section{Bronchoscopic procedures}

Most of the patients underwent general anesthesia. Propofol (Libang Pharmaceutical Co., Ltd., Xi'an, China) was used for sedation $(1.0 \mathrm{mg} / \mathrm{kg}$ for induction and $3.0-6.0 \mathrm{mg} / \mathrm{kg} / \mathrm{h}$ for maintenance), and remifentanil (Jiangsu Hengrui Medicine Co., Ltd., Lianyungang, China) was used for analgesia $(5.0-10.0 \mu \mathrm{g} / \mathrm{kg} / \mathrm{h}$ ). If needed, cisatracurium (Jiangsu Hengrui Medicine Co., Ltd., Lianyungang, China) was used for the induction of neuromuscular blockade at a dosage of 0.15 $\mathrm{mg} / \mathrm{kg}$. Patients were intubated with a laryngeal mask airway (Well Lead Medical Co., Ltd., Guangzhou, China) and ventilated using a closed circuit connected to a ventilator. In local anesthesia, after premedication with $0.5 \mathrm{mg}$ of atropine (orally) and 2\% lidocaine (Hubei Tianyao Pharmaceutical Co., Ltd., Xiangyang, China) (aerosol inhalation) within half an hour prior to the bronchoscopy, the procedures were performed by bronchoscopists using fiberoptic bronchoscopy (BF-1T60; Olympus Corp., Tokyo, Japan). All bronchoscopies were performed by two experienced bronchoscopists.

Biopsy methods included forceps biopsies and cryobiopsy. In general, three to five biopsies were performed by forceps biopsy ${ }^{9,14}$ and one or two biopsies by cryobiopsy. ${ }^{10,15}$ When endobronchial tumors bled significantly following the first biopsy attempt, only one biopsy was taken. Cold water, diluted adrenalin (Hangzhou Minsheng Pharmaceutical Co., Ltd., Hangzhou, China), and argon plasma coagulation (APC) were used for endobronchial hemostasis.

\section{Statistical analysis}

Statistical analysis was performed using SPSS 21.0 software (IBM Corporation, Armonk, NY, USA). Blood tests values were expressed as the mean \pm standard error of the mean and analyzed using a two-sided unpaired $t$-test. Age was indicated as median (range). Pearson $\chi^{2}$ or the Fisher's exact test was used for categorical variables. Variables that were considered clinically relevant and those with a $p$ value of $<0.05$ in the univariate analysis were included in the multiple logistic regression. Data were considered statistically significant when $p<0.05$.

\section{Results}

A total of 531 eligible patients were enrolled, and the patient demographics are presented in Table 1. The 162 patients 
Table I Patient demographics

\begin{tabular}{ll}
\hline Characteristics & Values \\
\hline Age (years), median (range) & $65(32-85)$ \\
Male gender, $\mathrm{n}(\%)$ & $418(78.7)$ \\
Patients with coexisting disease, $\mathrm{n}(\%)$ & \\
COPD & $36(6.8)$ \\
Hypertension & $131(24.7)$ \\
Diabetes & $28(5.3)$ \\
Heart disease & $13(2.5)$ \\
Location of lesion, $\mathrm{n}(\%)$ & \\
Central airways & $87(16.4)$ \\
Peripheral bronchi & $444(83.6)$ \\
Histologic types, $\mathrm{n}$ (\%) & \\
Adenocarcinoma & $141(26.6)$ \\
Squamous cell carcinoma & $268(50.5)$ \\
SCLC & $100(18.8)$ \\
NSCLC (not specified) & $6(1.1)$ \\
Neuroendocrine carcinoma & $4(0.8)$ \\
Adenosquamous carcinoma & $5(0.9)$ \\
Adenoid cystic carcinoma & $1(0.2)$ \\
Histology not specified & $6(1.1)$ \\
Stage, $\mathrm{n}$ (\%) & \\
Early & $313(59.0)$ \\
Advanced & $218(41.0)$ \\
Biopsy-induced bleeding, $\mathrm{n}$ (\%) & $162(30.5)$ \\
Methods of hemostasis, $\mathrm{n}$ (\%) & \\
Single cold water application & $81(50.0)$ \\
Combined application (cold water, adrenalin & $81(50.0)$ \\
or/and APC) & \\
\hline Abbrevatons: APC, ar & \\
\hline
\end{tabular}

Abbreviations: APC, argon plasma coagulation; NSCLC, non-small-cell lung carcinoma; SCLC, small-cell lung carcinoma.

who received treatment of endobronchial hemostasis were categorized as the bleeding group, in which $50 \%$ of the patients $(n=81)$ only needed intrabronchial instillation of 5-10 $\mathrm{mL}$ cold water (2-3 times repeated if needed) to stop bleeding, while the remaining patients required topical instillation of small amounts of diluted adrenalin $(1: 10,000)$ or APC to control bleeding after cold water treatment. In none of the cases, severe bleeding was observed. The other 369 patients, who did not need any maneuvers for hemostasis, were categorized as the non-bleeding group.

The location of the lesion, histologic type, stage, and C-reactive protein positively correlated with hemorrhage of EBB as assessed by univariate analysis (Table 2). However, only the location of the lesion, histologic type, and the stage of lung cancer were independently associated with EBB bleeding as assessed by multiple logistic regression (Table 3). Other variables, such as age, gender, coexisting illnesses with COPD, diabetes, hypertension, or heart disease, were not statistically significant between the bleeding group and the non-bleeding group $(p>0.05)$. In addition, none of the blood tests were associated with bleeding during $\mathrm{EBB}(p>0.05)$.
Table 2 Univariable analysis of risk factors for endobronchial biopsy-induced bleeding

\begin{tabular}{|c|c|c|c|}
\hline \multirow[t]{2}{*}{ Variables } & \multicolumn{2}{|c|}{ Biopsy-induced bleeding } & \multirow[t]{2}{*}{$p$-value } \\
\hline & Yes & No & \\
\hline Age, years (range) & $66(36-85)$ & $65(32-85)$ & 0.152 \\
\hline \multicolumn{4}{|l|}{ Gender, n (\%) } \\
\hline Male & $130(3||)$. & $288(68.9)$ & 0.569 \\
\hline Female & $32(28.3)$ & 81 (7I.7) & \\
\hline \multicolumn{4}{|l|}{ Diabetes, n (\%) } \\
\hline Yes & $7(25.0)$ & $21(75.0)$ & 0.515 \\
\hline No & $155(30.8)$ & $348(69.2)$ & \\
\hline \multicolumn{4}{|l|}{ Hypertension, n (\%) } \\
\hline Yes & $4 I(3 \mid .3)$ & $90(68.7)$ & 0.821 \\
\hline No & $121(30.3)$ & $279(69.7)$ & \\
\hline \multicolumn{4}{|l|}{ COPD, n (\%) } \\
\hline Yes & II (30.6) & $25(69.4)$ & 0.995 \\
\hline No & I5I (30.5) & $344(69.5)$ & \\
\hline \multicolumn{4}{|l|}{ Heart disease, n (\%) } \\
\hline Yes & $4(30.8)$ & $9(69.2)$ & 1.000 \\
\hline No & $158(30.5)$ & $360(69.5)$ & \\
\hline \multicolumn{4}{|l|}{ Location of lesion, n (\%) } \\
\hline Central airway & $45(5 \mathrm{I} .7)$ & $42(48.3)$ & 0.000 \\
\hline Peripheral bronchi & $117(26.4)$ & $327(73.6)$ & \\
\hline \multicolumn{4}{|l|}{ Histologic types, n (\%) } \\
\hline Adenocarcinoma & $22(15.6)$ & $119(84.4)$ & 0.000 \\
\hline Squamous cell carcinoma & $102(38.1)$ & $166(61.9)$ & \\
\hline SCLC & $33(33.0)$ & $67(67.0)$ & \\
\hline \multicolumn{4}{|l|}{ Stage, $n(\%)$} \\
\hline Early & $85(27.2)$ & $228(72.8)$ & 0.044 \\
\hline Advanced & $77(35.3)$ & I4I (64.7) & \\
\hline WBC $\left(\times 10^{9} / L\right)$ & $7.47 \pm 0.22$ & $7.31 \pm 0.16$ & 0.589 \\
\hline Neutrophils $\left(\times 10^{9} / L\right)$ & $5.47 \pm 0.21$ & $5.20 \pm 0.17$ & 0.327 \\
\hline CRP (mg/L) & $26.40 \pm 2.70$ & $20.04 \pm 1.65$ & 0.046 \\
\hline Hemoglobin, g/dL & $|2.78 \pm 0.2|$ & $12.80 \pm 0.18$ & 0.93 \\
\hline Plt $\left(\times 10^{9} / \mathrm{L}\right)$ & $245.02 \pm 8.22$ & $230.10 \pm 4.53$ & 0.113 \\
\hline PT (S) & $13.59 \pm 0.62$ & $16.34 \pm 1.30$ & 0.056 \\
\hline APTT (S) & $37.47 \pm 1.55$ & $39.32 \pm 1.46$ & 0.445 \\
\hline ALT (IU/L) & $19.31 \pm 1.25$ & $22.11 \pm 0.94$ & 0.088 \\
\hline AST (IU/L) & $25.40 \pm 1.24$ & $26.44 \pm 0.76$ & 0.466 \\
\hline
\end{tabular}

Abbreviations: ALT, alanine aminotransferase; APTT, activated partial thromboplastin time; AST, aspartate aminotransferase; CRP, C-reactive protein; PIt, platelet; PT, prothrombin time; SCLC, small-cell lung carcinoma; WBC, white blood cell.

During biopsy, the lesions located in the central airways were more susceptible to bleeding compared to those in peripheral bronchi (odds ratio [OR], 2.211; 95\% CI, 1.276-3.830; $p=0.005$; Figure 1). Moreover, no significant differences were found between the trachea, left main bronchus, and the right main bronchus (including the right middle bronchus) ( $p=0.42$, Table 4 ). In addition, no significant differences were observed between the left lobar bronchi and the right lobar bronchi ( $p=0.19$, Table 4 ). The upper lobar bronchi and the lower lobar bronchi were not significantly different in biopsy-induced bleeding ( $p=0.05$, Table 4 ). 
Table 3 Multivariable analysis of risk factors for endobronchial biopsy-induced bleeding

\begin{tabular}{llll}
\hline Variables & Odds ratio & $\mathbf{9 5 \%} \mathbf{C l}$ & p-value \\
\hline CRP & 1.003 & $0.997-1.009$ & 0.315 \\
$\begin{array}{l}\text { Location of lesion } \\
\quad \text { Peripheral bronchi }\end{array}$ & & & \\
$\quad$ Central airway & 2.211 & $1.276-3.830$ & 0.005 \\
$\begin{array}{l}\text { Histologic types } \\
\quad \text { Adenocarcinoma }\end{array}$ & & & \\
$\quad$ Squamous cell carcinoma & 3.107 & $1.832-5.27 \mathrm{I}$ & 0.000 \\
$\quad$ SCLC & 2.389 & $1.271-4.489$ & 0.007 \\
$\begin{array}{l}\text { Stage } \\
\quad \text { Early }\end{array}$ & & & \\
$\quad$ Advanced & 1.583 & $1.065-2.354$ & 0.023 \\
\hline
\end{tabular}

Abbreviations: CRP, C-reactive protein; SCLC, small-cell lung carcinoma.

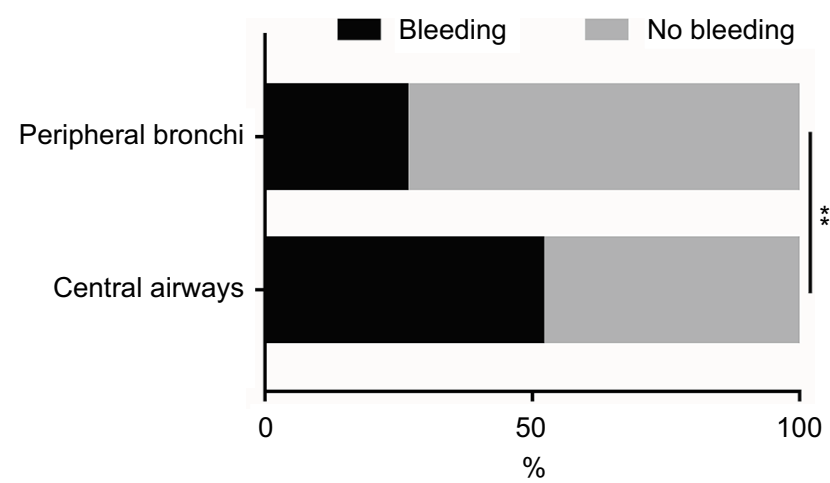

Figure I The incidence rates of bleeding of lesions located in the central airways and peripheral bronchi during EBB.

Notes: The incidence rate of biopsy-induced bleeding was higher in lesions located in the central airways compared to that in lesions located in the peripheral bronchi. $* * p<0.01$.

Abbreviation: EBB, endobronchial biopsy.

Table 4 Locations of lesion of endobronchial biopsy-induced bleeding

\begin{tabular}{|c|c|c|c|}
\hline \multirow[t]{2}{*}{ Locations of lesion } & \multicolumn{2}{|c|}{$\begin{array}{l}\text { Biopsy-induced } \\
\text { bleeding }\end{array}$} & \multirow[t]{2}{*}{$p$-value } \\
\hline & Yes & No & \\
\hline \multicolumn{4}{|l|}{ Central airway, n (\%) } \\
\hline Trachea & $4(40)$ & $6(60)$ & 0.42 \\
\hline Left main bronchus & $20(60.6)$ & $13(39.4)$ & \\
\hline $\begin{array}{l}\text { Right main and right middle } \\
\text { bronchi }\end{array}$ & $21(47.7)$ & $23(52.3)$ & \\
\hline \multicolumn{4}{|l|}{ Peripheral bronchi, n (\%) } \\
\hline Left lobar bronchi & $63(29.2)$ & I53 (70.8) & 0.19 \\
\hline Right lobar bronchi & $54(23.7)$ & $174(76.3)$ & \\
\hline Upper lobar bronchi & $74(30.0)$ & $173(70.0)$ & 0.05 \\
\hline Lower lobar bronchi & $37(21.4)$ & $136(78.6)$ & \\
\hline
\end{tabular}

Considering the histologic types, squamous cell carcinoma (SCC; OR, 3.107; 95\% CI, 1.832-5.271; $p=0.000$ ) and small-cell lung carcinoma (SCLC; OR, 2.389; 95\% CI, $1.271-4.489 ; p=0.007)$ were more prone to bleeding during EBB when compared to adenocarcinoma (Figure 2). However,

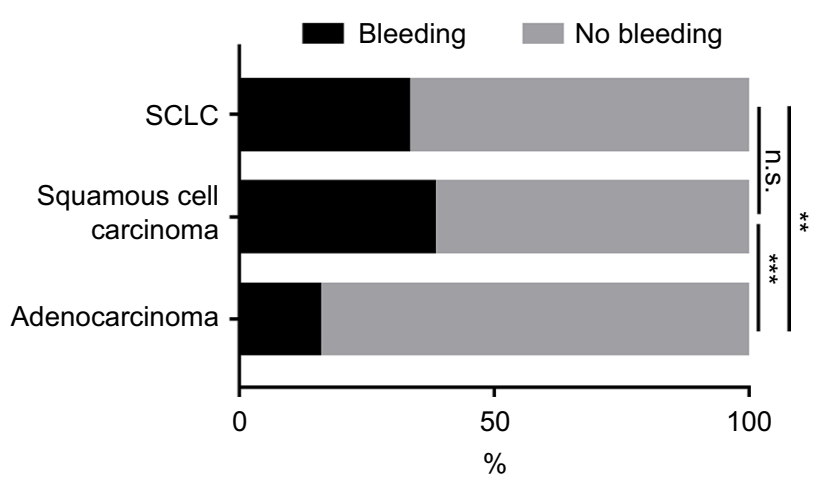

Figure 2 The incidence rates of bleeding among SCC, SCLC, and adenocarcinoma during EBB.

Notes: SCC and SCLC were more susceptible to bleeding when compared with adenocarcinoma, and it was not statistically significant between SCC and SCLC. n.s., $p>0.05, * * p<0.01, * * * p<0.001$.

Abbreviations: $\mathrm{EBB}$, endobronchial biopsy; n.s., not significant; SCC, squamous cell carcinoma; SCLC, small-cell lung carcinoma.

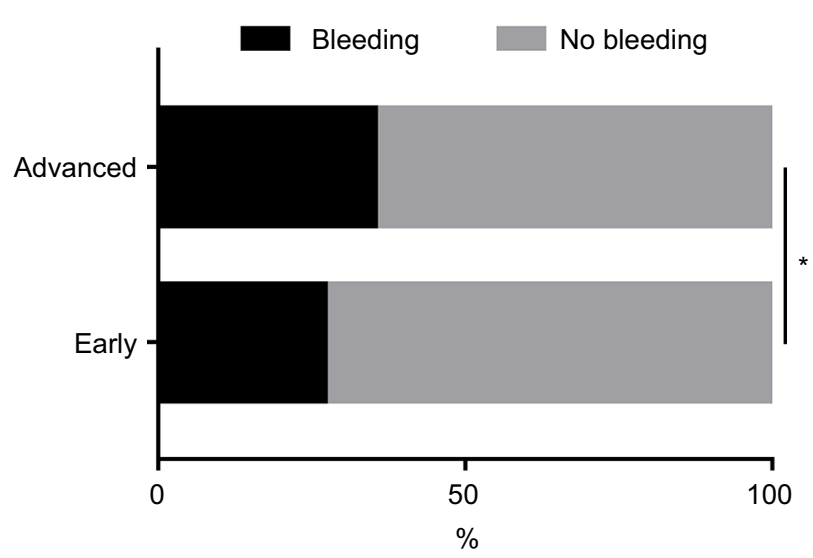

Figure 3 The incidence rates of bleeding in different stages of lung cancer during EBB.

Notes: The biopsy-induced bleeding events were more frequent in patients with advanced lung cancer compared to patients in the early stages of disease. ${ }^{*} p<0.05$. Abbreviation: EBB, endobronchial biopsy.

no statistically significant differences were observed between SCC and SCLC ( $p=0.298$, Figure 2$)$.

In addition, patients with advanced lung cancer were more likely to bleed upon EBB when compared to patients in the early stages of disease (OR, 1.583; 95\% CI, 1.065-2.354; $p=0.023$; Figure 3 ).

\section{Discussion}

In the present study, we demonstrated that the location, histologic type, and the stage of lung cancer were independent risk factors for bleeding during EBB. Moreover, we found that malignant lesions located in the central airways were more prone to bleeding compared to those found in peripheral bronchi. When compared with adenocarcinoma, SCC and SCLC were more susceptible to bleeding. In addition, it was more likely for bronchoscopists to encounter biopsy-related 
bleeding in patients with advanced lung cancer compared to patients in the early stages of disease.

The bronchoscopic procedure risk significantly increased when a biopsy was performed. ${ }^{1}$ The complication of biopsy-induced bleeding is very challenging for a bronchoscopist to manage. ${ }^{1-3}$ Reported rates of hemorrhage during bronchoscopy varied from $<1 \%$ to $\sim 20 \% .{ }^{4}$ When compared to $\mathrm{EBB}$, more common and more severe hemorrhage was reported when transbronchial biopsies were performed. It was noted that the rate of hemorrhage reached $26 \%$ during transbronchial biopsies. ${ }^{6}$ In general, when a larger sample size is obtained or a greater number of biopsy samples are taken, the risk of bleeding may increase. ${ }^{16}$ We obtained a larger sample size by using cryobiopsy; however, no significant differences were found in biopsy-induced bleeding between endobronchial forceps biopsies and cryobiopsy, ${ }^{10}$ both of which are widely used in the histopathologic diagnosis of lung cancer. ${ }^{9,10}$

In several studies, it was suggested that the risk factors for bleeding during bronchoscopy include immunosuppression, mechanical ventilation, thrombocytopenia (platelets $<50 \times 10^{3} / \mu \mathrm{L}$ ), pulmonary arterial hypertension, anticoagulant and antiplatelet therapy, liver and kidney disease, lung transplant, bleeding tendencies, and active bleeding. ${ }^{4}{ }^{-7}$ However, most patients who require bronchoscopy and subsequent biopsy do not have these serious "proposed risk factors". Malignancy, especially when accompanied by necrotic or various hypervascular tumors, is more likely to bleed during forceps biopsy or brush biopsy. ${ }^{11,12}$ In some malignant cases, massive blood loss following EBB may occur. ${ }^{17}$ However, lung cancer patients who are most prone to bleeding during EBB remain elusive.

In different populations, the risk factors for biopsyinduced bleeding may be inconsistent. ${ }^{4}$ In a Spanish retrospective study, including 1079 nonspecific study cases, it was reported that performing biopsies of the upper lobes involved risk factors that were associated with complications (hemorrhage, pneumothorax, desaturation $<80 \%$, and other complications). ${ }^{18}$ Moreover, Dreisin et $\mathrm{al}^{12}$ noted that SCC of the trachea in one of four patients developed significant bleeding following bronchoscopy. Carr et al ${ }^{19}$ enrolled 234 subjects who had a low clinical risk of bleeding and observed the presence of superior vena cava syndrome only was associated with bleeding during bronchoscopic biopsy.

Patients who showed "proposed risk factors" were excluded from our study. Indeed, most of the patients who underwent bronchoscopy did not show these risk factors in clinical practice. Therefore, patients enrolled in our study may represent a wider lung cancer study population.

We found that EBB-induced bleeding occurred more frequently in lesions located in the central airways compared to those in peripheral bronchi (bleeding rate, $51.7 \%$ vs. $26.4 \%$ ). However, neither biopsies performed on lesions in the upper lobar bronchi nor those performed on lesions in the left lobar bronchi were risk factors associated with biopsy bleeding compared to those performed on lesions in the lower or right lobar bronchi, respectively. SCC and SCLC were the pathologic types that were prone to bleeding during EBB. In addition, advanced stages of lung cancer were independent risk factors for EBB bleeding. The presence of coexisting illness, such as hypertension, COPD, diabetes, or heart disease (anticoagulant or antiplatelet therapy, preoperative stop for 7 days), did not predispose to bleeding. Nevertheless, given the rarity of the study on the population of coexisting diseases upon biopsy bleeding, ${ }^{4}$ future investigations are warranted to verify the data and to make a definite conclusion.

In this study, the strengths included having two fixed experienced bronchoscopists and a relatively fixed number of biopsies, thereby avoiding a potential operator-associated bias. However, in our study, we reported a significantly high proportion of bleeding cases $(30.51 \%, 162 / 531)$. Reportedly, persistent endobronchial bleeding was defined as the need for continuous suctioning for $\geq 2$ minutes. ${ }^{20}$ In our study, the 531 patients enrolled were categorized into two groups based on whether they had been treated for hemorrhage during biopsy. It is possible that several cases that were divided into the bleeding group would have spontaneously resolved biopsy-induced bleeding within 2 minutes. However, in clinical practice, we do not wait for 2 minutes to perform the hemostasis maneuvers. This may in part be contributed to the high bleeding rate. Another possible cause of the high bleeding rate is the nature of the study population, in which lung cancer was confirmed in all patients. It was reported that malignant lesions are more prone to bleed upon biopsy when compared to benign mucosal lesions. ${ }^{11-13}$

\section{Limitations}

Our study was subject to the potential limitations of the use of observational data. There were several potential confounding variables that would need to be reconciled. We did not further analyze the histologic types of non-small-cell lung carcinoma (not specified), adenosquamous carcinoma, neuroendocrine carcinoma, adenoid cystic carcinoma, and cases without histologic classification because the number of samples 
was too small (Table 1). Therefore, statistical results of the histologic types of lung cancer were not exact. Since adenocarcinoma, SCC, and SCLC account for the majority of lung cancer, we performed statistical analyses in cases of these three histologic types and observed a clinical significance. Hemorrhage is often only determined by direct observation during bronchoscopy. Schumann et $\mathrm{al}^{21}$ defined the amount of hemorrhage that occurred during biopsy procedures as follows: no bleeding or minimal bleeding (if bleeding stopped on its own), mild bleeding (if cold water or adrenalin solution was required), moderate bleeding (if APC or bronchial balloon blockage was required), and severe bleeding (events with hemodynamic instability). During bronchoscopy, it is challenging to accurately measure blood loss. Reasons are insensible bleeding into the bronchial tree and inaccurate estimation of the volume of blood that is aspirated. ${ }^{21}$ In our study, we categorized patients into a bleeding group and a non-bleeding group based on whether they had been treated with hemostasis maneuvers during biopsy. Therefore, a quantitative measurement of the volume of bleeding was also not provided.

Despite some potential limitations, our study was the first to identify the risk factors of EBB-induced bleeding in the lung cancer population without "proposed risk factors".

\section{Conclusion}

Patients with tumor lesions located in the central airways, histologic types of SCC and SCLC, and advanced stages may be considered as a "susceptible to bleeding" population during EBB. Knowing the risk factors of bleeding is critical for preventing and managing hemorrhage. Judicious selection of patients undergoing biopsy from this "susceptible to bleeding" population may be effective to reduce the biopsy-induced bleeding rate. ${ }^{1}$ Several hemostasis methods, including preoperative intrabronchial instillation of epinephrine or tranexamic acid ${ }^{22}$ to prevent biopsy bleeding, may be more specific when these risk factors are considered.

\section{Acknowledgments}

This study was supported by the Medical and Health Science and Technology Plan Project of Zhejiang Province (No. 2018263197 to SW) and the Youth Research Fund of Jinhua Hospital of Zhejiang University (No. JY2017205 to SW).

\section{Disclosure}

The authors report no conflicts of interest in this work.

\section{References}

1. Chinsky K. Bleeding risk and bronchoscopy: in search of the evidence in evidence-based medicine. Chest. 2005;127(6):1875-1877.

2. Bolliger CT, Sutedja TG, Strausz J, Freitag L. Therapeutic bronchoscopy with immediate effect: laser, electrocautery, argon plasma coagulation and stents. Eur Respir J. 2006;27(6):1258-1271.

3. Wahidi MM, Rocha AT, Hollingsworth JW, Govert JA, Feller-Kopman D, Ernst A. Contraindications and safety of transbronchial lung biopsy via flexible bronchoscopy. A survey of pulmonologists and review of the literature. Respiration. 2005;72(3):285-295.

4. Cordasco EM Jr, Mehta AC, Ahmad M. Bronchoscopically induced bleeding-a summary of nine years' Cleveland clinic experience and review of the literature. Chest. 1991;100(4):1141-1147.

5. Herth FJ, Becker HD, Ern A. Aspirin does not increase bleeding complications after transbronchial biopsy. Chest. 2002;122(4): 1461-1464

6. Zavala DC. Pulmonary hemorrhage in fiberoptic transbronchial biopsy. Chest. 1976;70(5):584-588.

7. Diette GB, Wiener CM, White P Jr. The higher risk of bleeding in lung transplant recipients from bronchoscopy is independent of traditional bleeding risks: results of a prospective cohort study. Chest. 1999;115(2): 397-402.

8. Siegel RL, Miller KD, Jemal A. Cancer statistics, 2017. CA Cancer J Clin. 2017;67(1):7-30.

9. Rivera MP, Detterbeck F, Mehta AC. Diagnosis of lung cancer: the guidelines. Chest. 2003;123 (Suppl 1):129S-136S.

10. Aktas Z, Gunay E, Hoca NT, et al. Endobronchial cryobiopsy or forceps biopsy for lung cancer diagnosis. Ann Thorac Med. 2010;5(4): 242-246.

11. Surratt DM, Smiddy JF, Bruber B. Deaths and complications associated with fiberoptic bronchoscopy. Chest. 1976;69(6):747-751.

12. Dreisin RB, Albert RR, Rabley PA. Flexible bronchoscopy in the teaching hospital: yield and comparison. Chest. 1978;74(2):148-149.

13. Ozgül MA, Turna A, Yildiz P, Ertan E, Kahraman S, Yilmaz V. Risk factors and recurrence patterns in 203 patients with hemoptysis. Tuberk Toraks. 2006;54(3):243-248.

14. British Thoracic Society Bronchoscopy Guidelines Committee, a Subcommittee of Standards of Care Committee of British Thoracic Society. British Thoracic Society guidelines on diagnostic flexible bronchoscopy. Thorax. 2001;56(Suppl 1):1S-21S.

15. Segmen F, Aktaş Z, Öztürk A, et al. How many samples would be optimal for endobronchial cryobiopsy? Surg Endosc. 2017;31(3):1219-1224.

16. Murgu S, Colt H. Role of the pulmonologist in ordering post-procedure molecular markers in non-small-cell lung cancer: implications for personalized medicine. Clin Lung Cancer. 2013;14(6):609-626.

17. Jin F, Mu D, Chu D, Fu E, Xie Y, Liu T. Severe complications of bronchoscopy. Respiration. 2008;76(4):429-433.

18. Fernández-Bussy S, Labarca G, Zagolin M, et al. Complicaciones asociadas a la broncoscopía flexible: análisis de registro post-procedimiento [Immediate complications following flexible bronchoscopy: retrospective analysis of 1079 procedures]. Rev Med Chil. 2014;142(3):299-304. Spanish

19. Carr IM, Koegelenberg CF, von Groote-Bidlingmaier F, et al. Blood loss during flexible bronchoscopy: a prospective observational study. Respiration. 2012;84(4):312-318.

20. Tüller C, Tüller D, Tamm M, Brutsche MH. Hemodynamic effects of endobronchial application of ornipressin versus terlipressin. Respiration. 2004;71(4):397-401.

21. Schumann C, Hetzel M, Babiak AJ, et al. Endobronchial tumor debulking with a flexible cryoprobe for immediate treatment of malignant stenosis. J Thorac Cardiovasc Surg. 2010;139(4):997-1000.

22. Zamani A. Bronchoscopic intratumoral injection of tranexamic acid to prevent excessive bleeding during multiple forceps biopsies of lesions with a high risk of bleeding: a prospective case series. BMC Cancer. 2014;14(1):1-5. 
Cancer Management and Research

\section{Publish your work in this journal}

Cancer Management and Research is an international, peer-reviewed open access journal focusing on cancer research and the optimal use of preventative and integrated treatment interventions to achieve improved outcomes, enhanced survival and quality of life for the cancer patient.

The manuscript management system is completely online and includes

Submit your manuscript here: https://www.dovepress.com/cancer-management-and-research-journal

a very quick and fair peer-review system, which is all easy to use. Visit $\mathrm{http}: / / \mathrm{www}$.dovepress.com/testimonials.php to read real quotes from published authors. 